\title{
ASPECTOS ECONÓMICOS DEL AISLAMIENTO ACÚSTICO
}

\author{
(ECONOMIC ASPECTS OF SOUNDPROOFING)
}

\author{
Beatriz C. Amarilla. Arq. Investigador. \\ Comisión de Investigaciones Científicas \\ de la Provincia de Buenos Aires. \\ República Argentina (C.I.C.-FAU/UNLP)
}

Fecha de recepcion: $12 \cdot \mathrm{XI} \cdot 90$

343.3

\begin{abstract}
RESUMEN
El objetivo general de este trabajo fue el de analizar el comportamiento de la relación costo-aislamiento acústico en soluciones constructivas alternativas para muros interiores y entrepisos.

Este estudio técnico-económico comprende tres partes:

* Muros divisorios (ruidos aéreos).

- Entrepisos (ruidos de impacto).

* Soluciones especiales (muros de doble hoja, pisos flotantes, etc.)

Se llega a la conclusión que, en los paises en desarrollo, no siempre las mejores soluciones para la vivienda, desde el punto de vista acústico, son las de mayor costo. Conocer en profundidad los aspectos económicos de esta cuestión significa poder lograr una buena calidad con costos moderados, lo cual constituye una prioridad en este tipo de paises.
\end{abstract}

\section{SUMMARY}

The general objective of this study was to analyze the soundproofing/cost ratio with different building alternatives for interior walls and floors.

This technical-economic study was divided into three parts:

- Dividing walls (anvironmental noises)

- Floors (impact noices)

- Special solutions (double walls, floating floors, etcétera)

The results show that in developing countries the most costly solutions are not always the best for housing, as far as soundproofing is concerned. A good knowledge of the economic aspects related to this matter allows obtaining a good quality at a moderate cost, which is a priority in this type of Country.

\section{INTRODUCCIÓN}

La calidad objetiva en la construcción surge de aspectos que se relacionan con la habitabilidad, durabilidad y seguridad de los edificios. Dentro de este marco, puede afirmarse que el aislamiento acústico es uno de los factores que se suele tener menos en cuenta, especialmente en el diseño de viviendas colectivas.

Esta realidad obedece, por lo menos en la República Argentina, a varias causas, entre las que merecen citarse:

a) Escasa consideración de los aspectos nocivos del ruido en el desarrollo de las actividades de la vida cotidiana (fatiga nerviosa, efectos inhibitorios sobre el trabajo, aumento inconciente del esfuerzo, dificultad para la recuperación del potencial nervioso y energético, etc) (1). b) Reducido capital inicial para invertir en la construcción, lo que lleva a atender, en el mejor de los casos, otros aspectos de calidad prioritarios. Por ejemplo, en un territorio con climas tan variados como la República Argentina, el confort térmico no puede postergarse en las zonas bioambientales con climas extremos, muy cálidos a frios (zona noroeste, sur, etc.).

c) Adherir el concepto de que cuanto más aislamiento acústico se requiera, más costosa será la solución, especialmente a partir de ciertos niveles de exigencia. Esta idea, que suele ser válida, no puede aplicarse directamente a situaciones macroeconómicas atípicas o a economías en crisis.

Esta última hipótesis (el hecho de que en países en desarrollo no existe hoy un crecimiento proporcional de los costos y de la eficiencia del aislamiento acústico, 
originó un Proyecto de Investigación, algunos de cuyos resultados se exponen a continuación (2).

El objetivo general del trabajo fue analizar diferentes soluciones tecnológicas para muros interiores y entrepisos, para observar en qué casos se optimiza la relación costo-calidad, medida esta última desde el punto de vista del aislamiento acústico.

\section{AISLAMIENTO ACÚSTICO DE MUROS DIVISORIOS (RUIDOS AÉREOS)}

Las fuentes de información que se utilizaron en este caso fueron básicamente dos:

a) La Norma IRAM 4.044 (junio de 1985): "Protección contra el ruido en edificios. Aislamiento acústico mínimo de tabiques y muros". Dicha Norma especifica valores de $\mathrm{Rw}$ (indice de reducción acústica compensado) para diferentes tipos de edificios, y diversas soluciones constructivas para planos verticales interiores.

b) La Revista "Vivienda" (marzo de 1990), publicación mensual que contiene datos sobre costos de rubros de obra y materiales de construcción, en base a un modelo establecido de edificio de vivienda colectiva.

La figura 1 muestra la relación $\mathrm{Rw}(\mathrm{dB}) / \operatorname{Costo}\left(\mathrm{A} / 10 \mathrm{~m}^{2}\right)$ para los diferentes tipos de muros citados en la Norma IRAM 4.044. A ellos se agregó, por considerarlo de interés, el caso del ladrillo de roca de yeso, cuyos datos de Rw corresponden a ensayos realizados en el INTI (3).

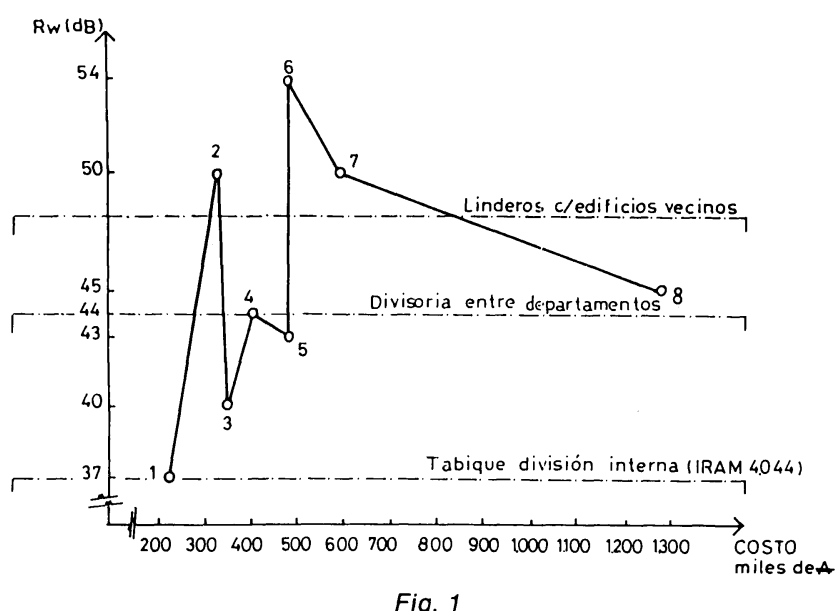

1. Lad. roca de yeso.- 2. Lad. común 0,12.-3. Lad. hueco 0,11.-

4. Lad. hueco 0,18. - 5. Placas de yeso.-6. Lad. común 0,28.-

7. $H^{\circ} A^{\circ}-8 . H^{\circ} A^{\circ}$ premoldeado.

(C) Consejo Superior de Investigaciones Científicas Licencia Creative Commons 3.0 España (by-nc)
La unidad de costo $A / 10 \mathrm{~m}^{2}$ se adoptó en base a los siguientes criterios:

- El tamaño superficial de una pared influye en el aislamiento acústico de la misma (a igual tecnología y espesor, un muro pequeño tiende a aislar más que uno más grande).

- La superficie normal de una pared divisoria entre dos ambientes de una vivienda suele ser de aproximadamente $10 \mathrm{~m}^{2}$.

- La Norma IRAM 4.063 sugiere que en ensayos en laboratorio para medir el aislamiento acústico, se utilicen muestras de pared de unos $10 \mathrm{~m}^{2}$, con un lado mínimo de 2,3 metros.

En el campo determinado por los ejes de coordenadas se sitúan las diferentes soluciones de muros estudiados. También aparecen los límites mínimos de aislamiento sugeridos por la Norma para muros con diferentes funciones (de división interna en la vivienda, medianera entre apartamentos, etc.).

A los efectos de comparar costo y calidad acústica puede tomarse como ejemplo a las soluciones 2 (ladrillo común de 0,12 metros de espesor) y 7 (tabique de hormigón armado). El valor de Rw es el mismo (50 dB) sin embargo, el costo de la segunda opción es casi el doble que el de la primera. Lógicamente, un muro tiene funciones múltiples (estructural, cerramiento, aislación térmica e hidrófuga, etc.), y por lo tanto el aislamiento acústico es sólo un aspecto de un problema más global.

Habrá que tener en cuenta que un concepto integral del costo no debería sólo considerar el proceso de construcción de la pared (materiales, equipos, mano de obra). Un muro pesado, comparado con una solución liviana, registra un costo suplementario porque necesita una capacidad adicional de soporte. Además, su mayor espesor indica que ocupa más superficie horizontal, por lo que, si se desea mantener invariable la superficie útil de los ambientes, el área en planta deberá crecer, con el consiguiente incremento en el costo (4).

Un concepto global del costo tampoco debería descuidar los aspectos macroeconómicos del problema. Por ejemplo, una buena solución constructiva desde el punto de vista acústico y de poco costo para el comitente podría estar utilizando alguna energía escasa en el país durante su proceso de producción, provenir de una fábrica contaminante, etc.

Los anteriores ejemplos indican que la definición del concepto de costo presenta cierta complejidad, sin contar el problema económico referido al uso y mantenimiento de los edificios, tema ya tratado en una publicación anterior (5).

http://informesdelaconstruccion.revistas.csic.es 

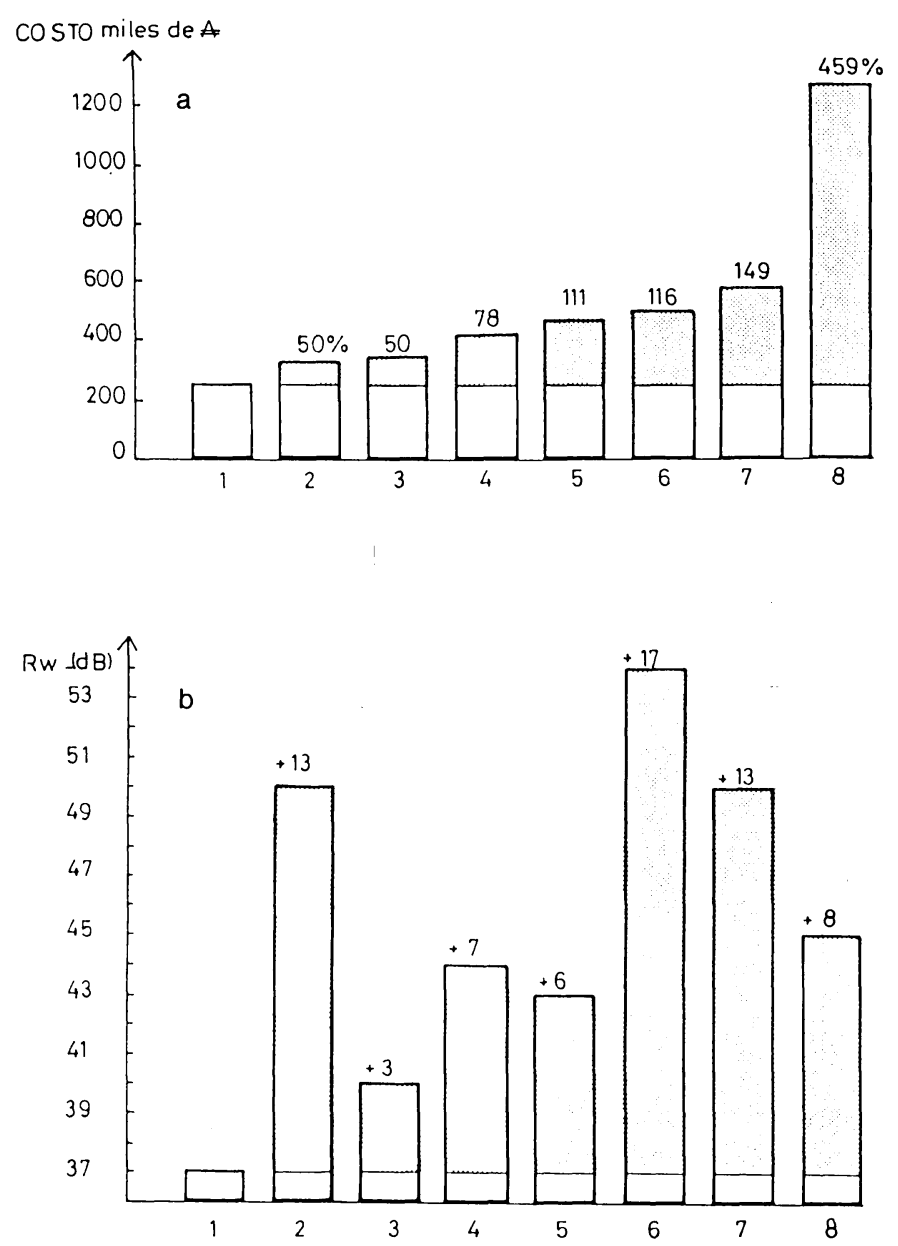

Fig. 2

La figura 2 a y $b$ muestra que los costos de las diferentes soluciones analizadas y su valor de Rw no aumentan en forma proporcional. El ejemplo 2 es el segundo en costo más bajo entre los ocho casos estudiados. Sin embargo, su performance acústica es igual o superior a la del resto de los ejemplos más caros. Sólo se exceptúa el caso 6 (también muro de ladrillo común, pero de 0,27 metros de espesor).

Las causas de estos resultados se relacionan con las propiedades físicas de los materiales, y con la coyuntura económica. Por una parte, por la "ley de masa", es sabido que a una frecuencia dada, el aislamiento acústico de una pared aumenta aproximadamente $4 \mathrm{~dB}$ cuando la masa se duplica y disminuye unos $4 \mathrm{~dB}$ cuando la masa se divide por dos (6). Ello explica que las opciones donde la masa superficial es elevada resulten adecuadas desde el punto de vista acústico.

Por otra parte, estos materiales en general son de origen artesanal, y no industrializado. Se ha demostrado que este tipo de materiales son baratos en los países en desarrollo: entre sus insumos el factor mano de obra tiene mucha incidencia en su producción y colocación; y se sabe que la mano de obra, especialmente la no especializada, tiene muy baja retribución en este tipo de países, si se comparan sus salarios con los de los empleados de otras industrias manufactureras (7).

\section{AISLAMIENTO ACÚSTICO DE ENTREPISOS (RUIDOS DE IMPACTO)}

Para el análisis de los entrepisos (forjados) se empleó una metodología similar a la anterior. En este caso la Norma correspondiente está aún en estudio. Se tomó como parámetro de medición la calidad a $\triangle \mathrm{Nw}$ (mejora en el índice de reducción del sonido de impacto compensado), o sea la diferencia entre los niveles de sonido de impacto compensado de un piso de referencia sin y con el recubrimiento de piso. Estos valores se han obtenido de resultados de ensayos de muestras realizados en laboratorio.

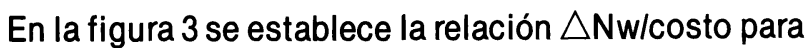
diferentes soluciones, entre las que se incluyen losas de hormigón armado y contrapisos flotantes, ambos con distintos tipos de recubrimiento de pisos.

El contrapiso flotante sin revestimiento, que aparece a la izquierda en el gráfico mencionado, no se ha usado con fines comparativos, ya que, por su terminación superficial, no es asimilable desde el punto de vista funcional al resto de soluciones alternativas analizadas. Los ejemplos 3 y 6 (contrapiso flotante con piso vinílico y de goma, respectivamente) muestran la gran incidencia que tiene en el costo el material de recubrimiento, para performances acústicas que son similares. En efecto, el costo entre ambas soluciones crece aproximadamente un $280 \%$, cifra notable en paises con ausencia de capital y especialmente en planes masivos de construcción, donde un prototipo de vivienda suele multiplicarse un número de veces importante.

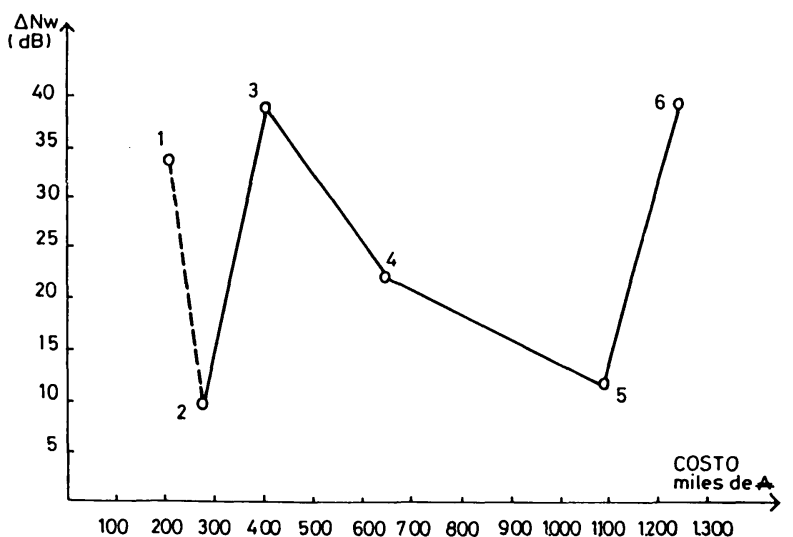

Fig. 3

1. Cont. flotante.- 2. Piso vinilico.- 3. $1+2 .-4$. Alfombra.5. Piso de goma. $-6.1+5$. 

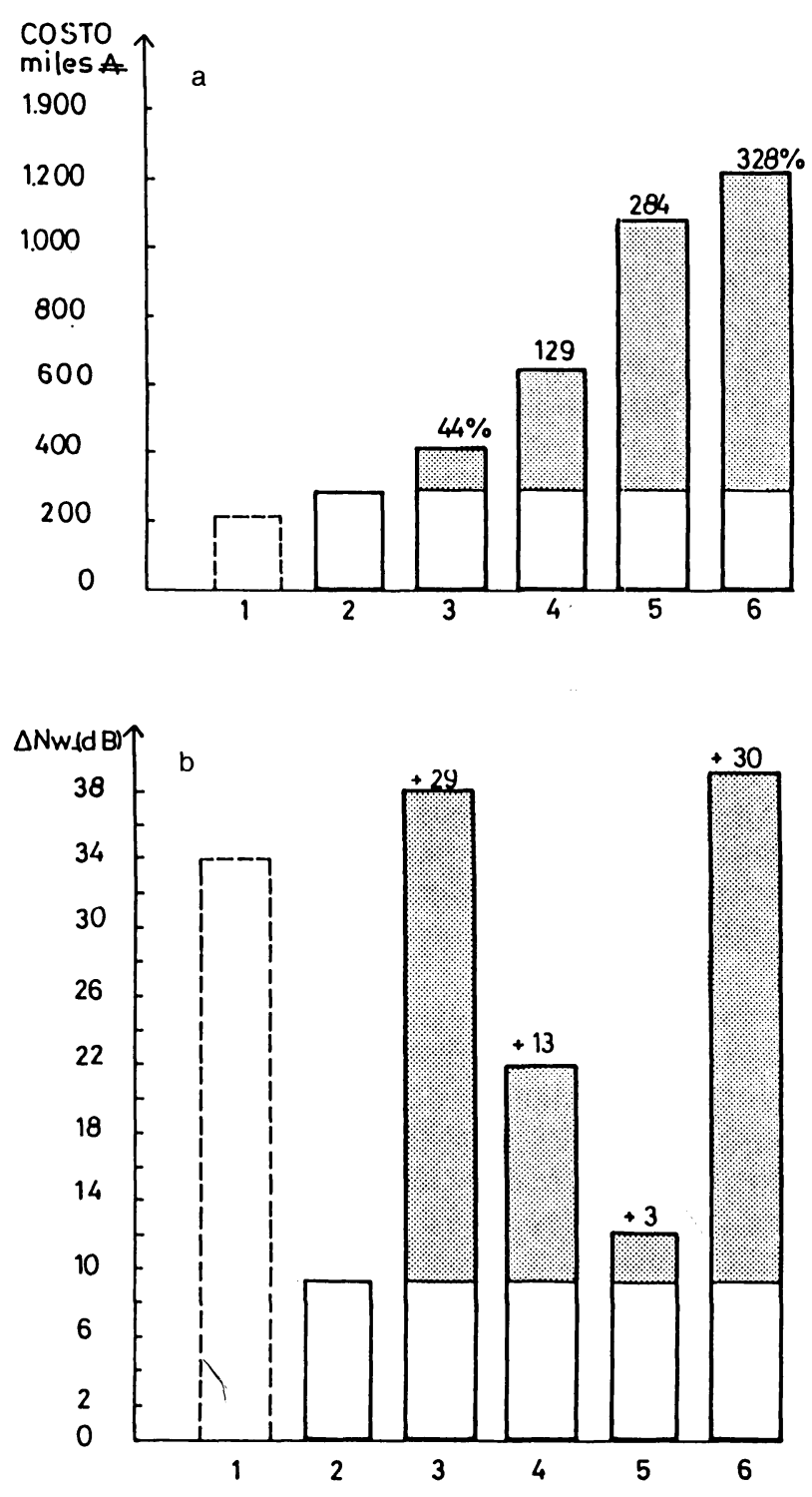

Fig. 4

La figura 4 a y b muestra la comparación entre el crecimiento del costo en las diferentes propuestas constructivas y la variación Nw correspondiente. Se observa que, descontando las soluciones con contrapiso flotante, una losa de hormigón armado común con revestimiento de alfombra tiene una buena performance acústica a los ruidos de impacto, con un costo que resulta moderado.

El crecimiento de costo en las alternativas analizadas tiene su causa, como ya se ha mencionado para el caso de los muros, en la producción artesanal o industrializada de los materiales, así como en el origen nacional o importado de ciertos insumos utilizados en su fabricación. En la figura 5 puede observarse la incidencia en el costo total de los materiales y de la mano de obra de colocación para cuatro alternativas de entrepisos. Hoy, por lo menos en la República Argentina, resulta que cuanto mayor incidencia en ese total tengan
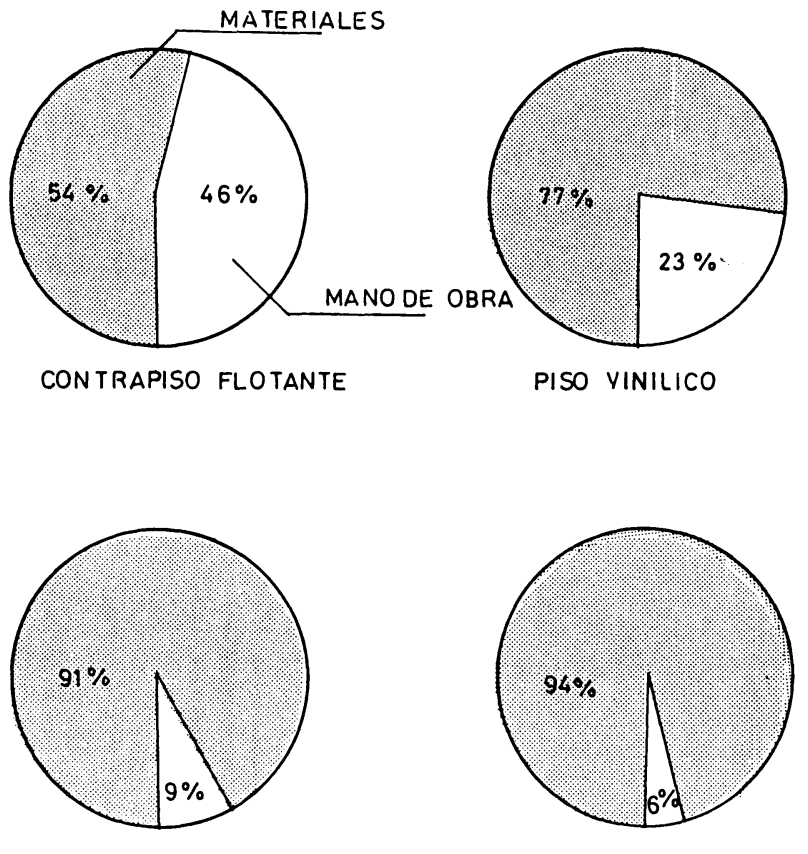

ALFOMBRA

PISO DE GOMA

Fig. 5 .

los materiales de construcción, mayor resultará el precio de venta del producto terminado. Históricamente, es uno de los momentos en que el peso porcentual de la mano de obra y de los materiales en el costo total ha alcanzado mayor desequilibrio, lo que se hace más evidente cuanto más industrializado sea el producto.

\section{ESTUDIO TÉCNICO-ECONÓMICO DE SOLUCIONES ESPECIALES}

Además de las ya analizadas, existen soluciones más especializadas, cuyo objetivo es lograr eficiencia respecto de ciertas exigencias concretas de los muros y entrepisos: es el caso, por ejemplo, de los pisos flotantes, muros de doble hoja, etc.

El éxito de estas soluciones radica, entre otros factores, en una correcta elección de materiales, una mano de obra eficiente y en un riguroso control técnico. Es posible suponer que ello traerá como consecuencia un incremento de costos; pero no se conoce de qué orden puede ser ese valor, y si se justifica en relación a las ventajas que se obtienen.

Dentro de estas soluciones especiales deben diferenciarse dos posibilidades:

- Cuando el elemento forma parte de la construcción original, y por lo tanto ha sido diseñado para cumplir con determinada función específica. 
- Cuando el elemento se introduce con posterioridad, con el objetivo de mejorar el nivel de aislamiento acústico original.

Según se presente alguna de estas dos situaciones, las soluciones posibles variarán, tanto en costo como en eficiencia. Las paredes pesadas, las losas o contrapisos flotantes son en general alternativas que se preven con el diseño del edificio. En cambio, los paneles livianos para muros y tabiques, los cielorrasos suspendidos, son tecnologías adecuadas para introducir mejoras posteriores, por su montaje en seco y facilidad de instalación.

Debido a la dificultad de evaluar económicamente a la mano de obra en el momento del análisis, se calculó, con carácter provisorio, sólo el costo de los materiales.

\section{a) Muros compuestos}

El aislamiento de una pared doble es muy diferente a la suma de los aislamientos de cada una de sus capas. Ambos elementos tienen un acople elástico a través de la cámara de aire. La vibración de una de las paredes se transmite a la otra a través de dicha capa elástica. La energía acústica transmitida por la primera pared golpea a la segunda; esta última transmite parte de esa energía al aire y parte la refleja, dando lugar a reflexiones múltiples en la cámara de aire (Fig. 6).

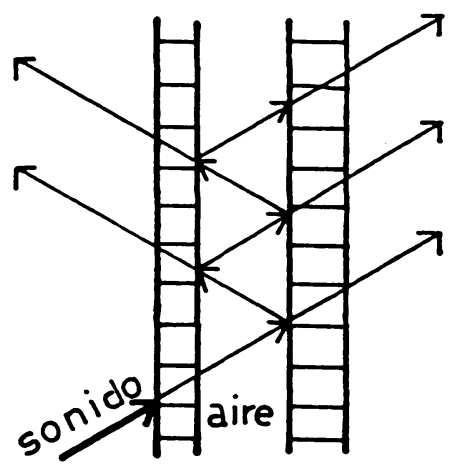

\section{PARED DOBLE}

Fig. 6

En las alternativas seleccionadas para su análisis, se han incluido una serie de variantes, las que se supone conducirán a un comportamiento acústico y económico muy diferente:

- Muros cuyas capas son de igual o diferente naturaleza (dos muros pesados; uno pesado y uno liviano; dos muros livianos).
- Existencia o no de un material absorbente intermedio (panel de fibra de vidrio de $25 \mathrm{~mm}$ de espesor. En ausencia de absorbente se estimó una cámara de aire de $3 \mathrm{~cm}$ en los ejemplos con absorbente; la cámara tiene $7 \mathrm{~cm}$ de espesor).
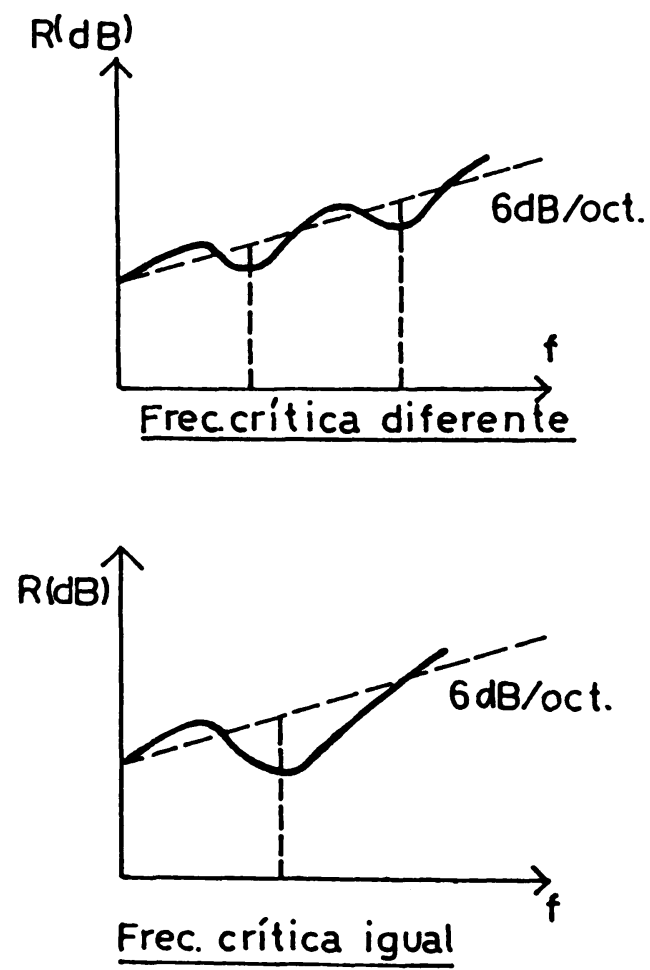

Fig. 7

Desde el punto de vista del aislamiento acústico, es importante que ambas hojas tengan diferente masa y rigidez. En las paredes simples, el aislamiento aumenta unos $4 \mathrm{~dB}$ cada vez que se duplica la frecuencia, con una disminución de dicho aislamiento en la llamada "Frecuencia crítica" del muro. En las paredes dobles el aislamiento aumenta de 6 a $8 \mathrm{~dB}$ por octava, y aqui la curva de aislamiento presenta varios defectos. Si dos paredes tienen frecuencias criticas diferentes, la curva de aislamiento presenta dos defectos. Si ambas paredes tienen igual frecuencia critica, vibran al unisono produciendo una resonancia, y el defecto de aislamiento resulta muy notable (Fig. 7).

La determinación del espesor de la cámara de aire es un factor esencial en relación al problema de las resonancias. Ese espesor debe ser tal que dichas resonancias se produzcan fuera de la zona sensible del oido (frecuencias superiores a $4.000 \mathrm{~Hz}$ ), o superiores a las frecuencias molestas del ruido que se desea aislar. Cuando las ondas sonoras son paralelas al muro, hay resonancias para frecuencias del sonido tales como: $17.000 \mathrm{~Hz} / \mathrm{d} ; 2 \times 17.000 \mathrm{~Hz} / \mathrm{d} ; 3 \times 17.000 \mathrm{~Hz} / \mathrm{d}$, donde "d" es justamente el espesor de la capa de aire intermedia entre ambas hojas. 
Las capas de material absorbente (fibra de vidrio, por ejemplo) son útiles desde varios puntos de vista:

- Atenúan la resonancia en la cámara de aire. El acoplamiento elástico entre ambas hojas se modifica, el absorbente consume parte de la energia sonora, lo que hace posible disminuir la caida de aislamiento a las frecuencias criticas de los elementos que componen la doble pared.

- Permite independizar ambas hojas de la pared, de manera que la unión entre ambas sea lo menos rígida posible. Cuando las uniones entre ambos elementos son numerosas (trabas metálicas, mortero colado, canalizaciones), la vibración de un elemento se comunica al otro. Así, la doble pared suele comportarse como un muro simple que tuviera dos frecuencias críticas en lugar de una sola (8).

Una vez comentadas estas generalidades sobre el significado acústico de las diferentes variantes consideradas, se puede pasar a su análisis económico.

Si se toma como referencia un muro de una sola capa de ladrillo común de $0,30 \mathrm{~m}$ de espesor, éste tiene un costo de materiales por metro cuadrado de alrededor de $A 13.000$ y una aislación de Rw de 54 dB. Es decir, es eficiente desde el punto de vista acústico, y su costo es menor al de cualquiera de las soluciones que se han analizado.

Esto significa que si la decisión se toma en el momento de construir el edificio, y el objetivo principal es obtener un buen aislamiento acústico, resulta preferible el uso de un muro único y pesado, y no soluciones de doble hoja con cámara de aire.

Si se observa el gráfico se puede deducir que los costos evolucionan de acuerdo al siguiente orden creciente:

- Muros tradicionales (pesados, semipesados).

- Un muro pesado y otro liviano.

- Dos muros livianos.

La inclusión de un material absorbente representa aproximadamente un crecimiento del costo del $25 \%$ res. pecto de la misma solución sin dicho elemento.

Debe tenerse en cuenta que un muro de doble hoja puede tener otros objetivos (aislamiento térmico, pasaje de canalizaciones, etc.). Además, un muro pesado puede representar, como se ha visto al principio, un mayor costo de soporte, mayor ocupación de superficie horizontal, etc. Ello significa, como ya se ha anticipado, que una óptima opción costo-calidad deberá tener en cuenta no sólo la exigencia prioritaria del muro, sino tam. bién el resto de usos o finalidades del mismo.

\section{b) Entrepisos}

En el caso de las losas o entrepisos de edificios, el concepto de pared doble puede adoptar alguna de las siguientes formas:

- Piso flotante: se basa en el uso de una losa armada (losa flotante) o un contrapiso (contrapiso flotante), separados de la estructura horizontal y de los cerramientos laterales mediante un material absorbente (lana de vidrio, poliestireno expandido, etc.), (Fig. 8).

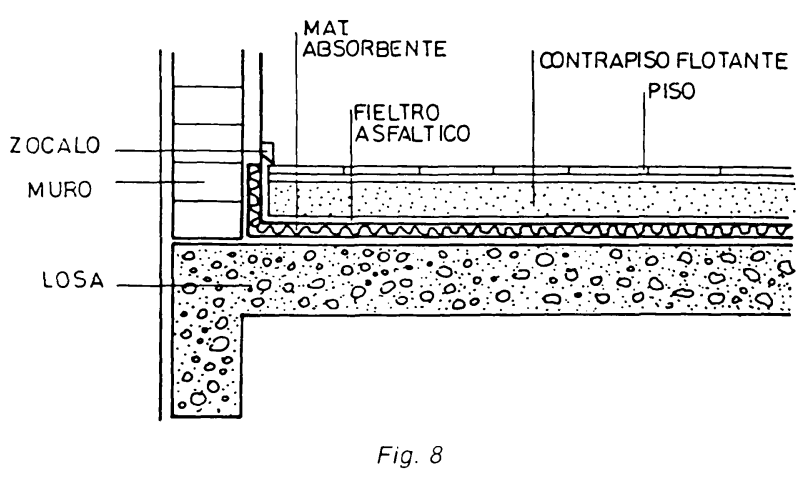

- Falso cielorraso: consiste en la colocación de un cielorraso de una masa superficial no inferior a 24 $\mathrm{kg} / \mathrm{m}^{2}$, separado de la estructura horizontal, a una distancia minima de $20 \mathrm{~cm}$. Su espesor debe ser superior a los $7 \mathrm{~cm}$ (Fig. 9).

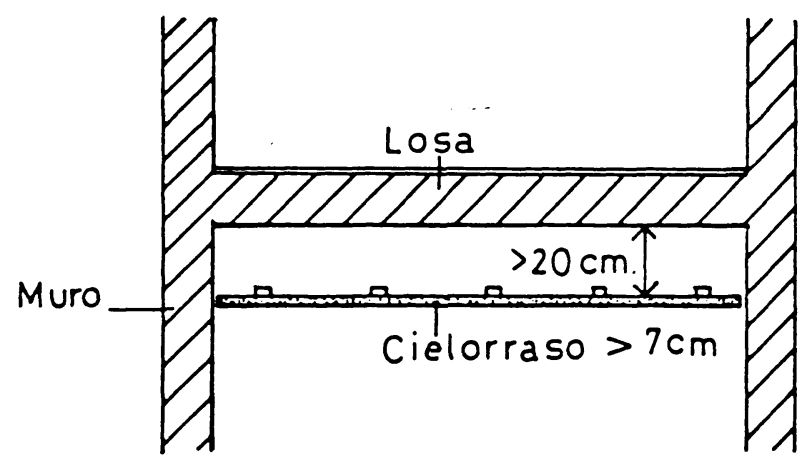

Fig. 9

- Cielorraso suspendido: se consigue colgando en forma elástica, de la estructura horizontal, un cielorraso, mediante dispositivos que aseguren dicho efecto. La separación entre losa y cielorraso debe ser de por lo menos 10 a $15 \mathrm{~cm}$ y es conveniente la colocación de un material absorbente en el espacio intermedio resultante (Fig. 10). 


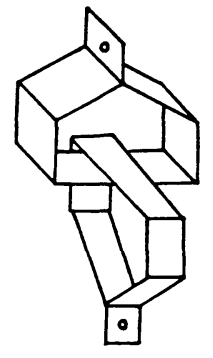

Mecanismo de suspensión

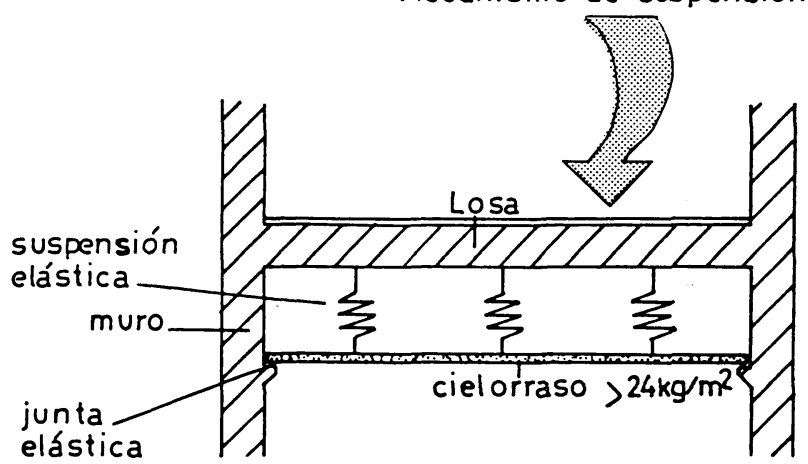

Fig. 10

Para la estimación de costos de estas alternativas, se ha previsto un revestimiento de piso uniforme (baldosas vinílicas), adicionando un porcentaje de costo en concepto de materiales complementarios (juntas, suspensiones elásticas, etc.)

En la figura 11 se ha graficado el costo de las diferentes soluciones analizadas. Se han dispuesto en orden creciente de costo, indicando el porcentaje de aumento que se registra por sobre la alternativa que resulta de costo menor.

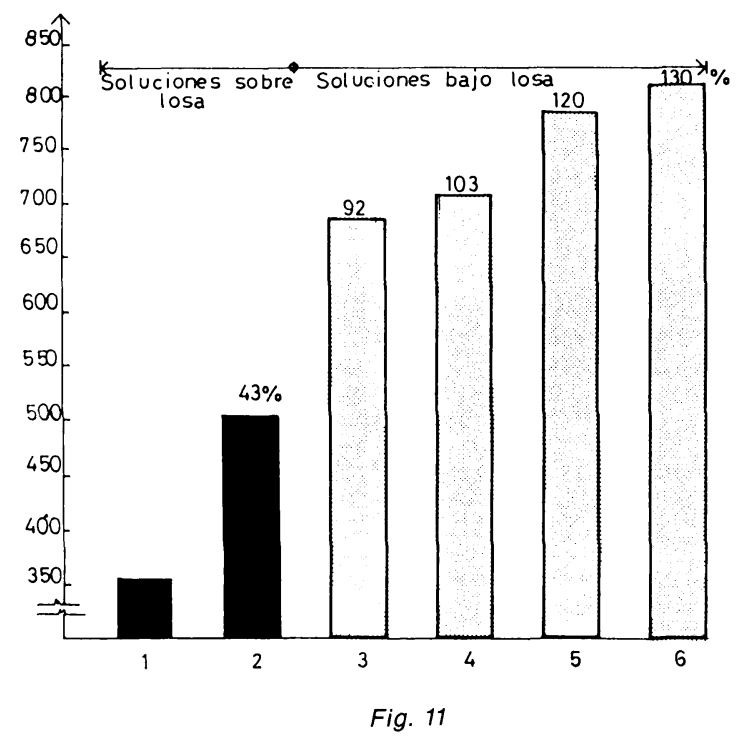

1. Contrapiso flotante.- 2. Losa flotante.- 3. Falso cielorraso.4. Idem $3 \mathrm{cl}$ absorbente.- 5 . Cielorraso suspendido.-

6. Idem $5 \mathrm{cl}$ absorbente.
Se aprecia que existen soluciones "por encima" de la losa, las que lógicamente serán más eficientes para atenuar los ruidos de impacto, ya que actúan antes de que los ruidos Ileguen a la estructura. El resto de alternativas se aplican "por debajo" de la losa, por lo que su eficiencia es sólo parcial, pues existirán ruidos que se transmiten lateralmente por la estructura.

Como resultado de la comparación costo-calidad, se observa que en este caso las soluciones "sobre losa", que por definición son las más aptas, son también actualmente las que presentan menores costos de materiales.

Se verifica lo antes afirmado para muros, en el sentido que las correcciones que es posible efectuar en edificios en funcionamiento tienen una relación costo-calidad que difícilmente pueda ser óptima. En este sentido el cambio de ciertos revestimientos de piso (por ejemplo, algunos tipos de alfombra) puede ser útil para amortiguar ruidos de impacto con costos moderados, y un fácil montaje que no altere demasiado las funciones de un edificio en uso.

\section{CONCLUSIONES}

La metodología empleada en este análisis puede en realidad hacerse extensiva, con pequeñas modificaciones, a todo estudio donde se pretenda relacionar las variables costo y calidad. El considerar estos dos factores como si fuesen términos de una misma ecuación se basa en un concepto clave: el costo de un material es una propiedad tan importante como su capacidad de aislar o absorber ruidos.

En efecto, muchos materiales y técnicas constructivas suelen ser descritos de tal manera que parecen tener propiedades excelentes y amplia aplicación. Pero ello queda restringido al plano teórico ya que los costos resultan excesivos, y sólo pueden así ser útiles en casos muy puntuales (9).

En sintesis, lo que se desea poner en evidencia es que todo proyectista, al elegir alternativas morfológicas y tecnológicas para responder a cada necesidad funcional, está haciendo, en forma consciente o no, directa o indirectamente, también una elección de costos de producción, montaje y mantenimiento.

El costo global de los edificios se ve afectado por el hecho de prever o no un adecuado aislamiento acústico. Estudios realizados indican que el aumento de costo de construcción, que tenga en cuenta el problema del ruido desde la etapa de proyecto, es de un 2 a un $4 \%$ en el caso de viviendas (10). En cambio, lograr mejoras sustanciales en edificios en uso necesita de intervenciones técnicamente complejas, e implica gas- 
tos importantes, difíciles de afrontar en paises que, como la República Argentina, tienen una restricción de capital muy significativa.

Si no existe un equilibrio entre costo y calidad, habrá un derroche o mal empleo del poco capital disponible. El mismo podría haber sido utilizado en obtener mayores ventajas cualitativas (más performance) y/o cuantitativas (más unidades de vivienda, por ejemplo).

Esta relación costo-calidad es siempre dinámica, y debe ser analizada en cada ocasión desde el punto de vista tanto macro como microeconómico. Así se da la paradoja, como se demuestra en los ejemplos estudiados, para un momento y lugar determinados, que ciertas soluciones muy efectivas no resultan hoy de costo excesivo. Esta situación evidentemente se modificaría si, como es de esperar, la relación salario-costo de vida evoluciona hacia valores más favorables. Ello indica la necesidad de que los proyectistas tengan presente la relación costo-performance al optar entre distintas alternativas de diseño, y que conozcan la dinámica de dicha relación según las características espacio-temporales del análisis que se está efectuando.

\section{AGRADECIMIENTOS}

Este trabajo se ha podido desarrollar gracias al asesoramiento en materia de aislamiento acústico que ha prestado el Laboratorio de Acústica y Luminotecnia de la Co- misión de Investigaciones Científicas de la Provincia de Buenos Aires. Agradezco la esmerada colaboración recibida, en especial de su Director, Ing. Antonio Méndez.

\section{REFERENCIAS}

1. Niilus, Malle R. V.: "Aislación acústica en la vivienda". Bouwncentrum Argentina. Buenos Aires, 1965.

2. Amarilla, B. C.: "Aislación acústica en viviendas colectivas: estudio de alternativas de costo y calidad". Comisión de Investigaciones Cientificas de la Provincia de Buenos Aires, 1989/90.

3. EI INTI es el Instituto Nacional de Tecnología Industrial. Los datos citados se extrajeron del Informe N. 37, mayoljunio de 1988.

4. Waller, R.: "Economics of sound reduction in buildings". Applied Acoustics Vol. 1, N. 3, Essex, 1968.

5. Amarilla, B. C.: "Los costos de mantenimiento de edificios en países en vias de desarrollo". Revista Informes de la Construcción N. 404, noviembre/diciembre de 1989.

6. Méndez, A.: "Aislación acústica". Revista Telegráfica Electrónica N. 778, Buenos Aires, 1977.

7. Mascaró, J. L.: “La construcción en la economía nacional". Facultad de Arquitectura y Urbanismo de la Universidad Nacional de La Plata, 1983.

8. Méndez, A.: “Aislación acústica". Revista Telegráfica Electrónica N. 780 y 781 . Buenos Aires, 1977/78.

\section{publicaciones del ICCET/CSIC}

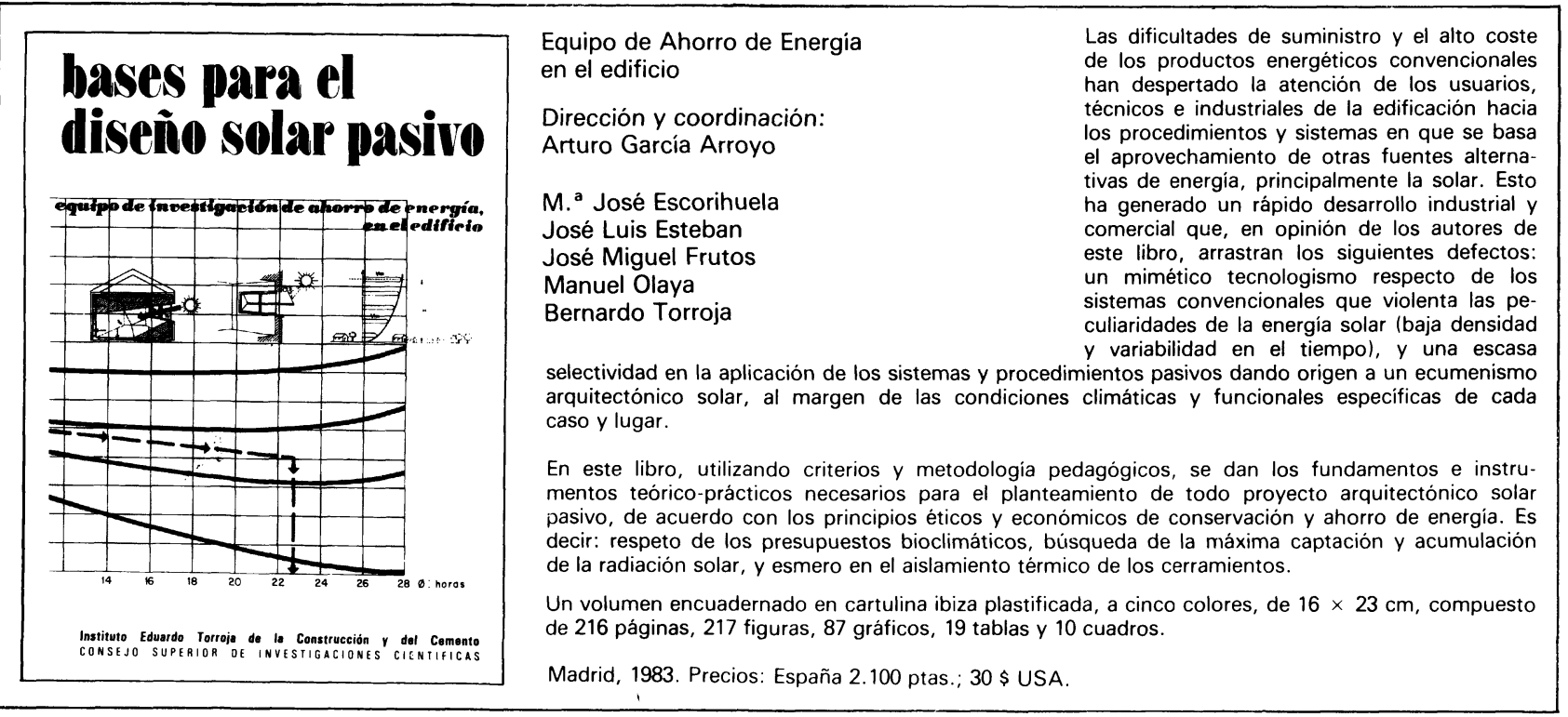

